\section{Response to: 'Time to personalise the treatment of anti-MDA-5 associated lung disease' by Lake et al}

We have with interest read the comments raised by Lake et $a l$ in the e-letter titled 'Time to personalise the treatment of anti-MDA-5 associated lung disease' to be published in your journal. ${ }^{1}$ We thank the authors for sharing their insights on the 2017 European League Against Rheumatism (EULAR)/American College of Rheumatology (ACR) classification criteria for adult and juvenile idiopathic inflammatory myopathies (IIM) and their major subgroups. ${ }^{2}$ Lake and colleagues are concerned that these classification criteria are not including rare specific subsets such as dermatomyositis (DM) or amyopathic DM associated with antimelanoma differentiation-associated protein 5 (MDA5). They fear that this will in turn prevent clinicians and researchers from exploring new therapeutic approaches for this population frequently affected by rapidly progressive interstitial lung disease (RPILD), a complication associated with fatal outcomes. ${ }^{3}$ Dr Malaviya raised a similar concern when discussing the importance of myositis-specific autoantibodies (MSA) in classifying subgroups of IIM, exemplified by four subgroups of amyopathic DM associated with different MSA and with different clinical phenotypes and response to treatment, one being anti-MDA5 antibody positive amyopathic DM. ${ }^{5}$ We responded to Dr Malaviya's concern and addressed the ongoing international collaborative effort of systematic collection of autoantibody and clinical data for patients with IIM. ${ }^{6}$ In this letter, we would like to address the concern raised by Lake and colleagues. Classification criteria and their influence on population selection for clinical trials surely have an impact on formulating treatment guidelines in conditions such as idiopathic inflammatory myositis (IIM). Classification of a heterogeneous group of diseases with multiple possible organ involvement, considerable overlapping features and different underlying pathological molecular mechanisms is a challenge. The 2017 EULAR/ACR IIM classification criteria were developed using the expertise of rheumatologists, dermatologists, neurologists and paediatricians in an attempt to reflect as closely as possible the clinical spectrum of IIM. As those criteria are data-driven, certain clinical characteristics such as most of the MSA could not be included in the final variables given their rarity and the fact that some of these MSA were not widely available for clinical use at time of data collection. However, the inclusion of anti-histidyl-tRNA synthetase (Jo1) in the current version of the criteria is a step forward in incorporating MSA as important discriminating factors in IIM classification. By no means are we suggesting to clinicians or researchers that MSA status do not have a diagnostic and prognostic utility in practice. It seems appropriate to emphasise that classification criteria should not be used as diagnostic criteria and that clinicians should consider all available evidence pointing to an IIM diagnosis to tailor their management. ${ }^{7}$

As emphasised by Lake et al, ILD is a serious and possibly fatal extramuscular manifestation of IIM. We agree that revision of the EULAR/ACR criteria should address this concern, as ILD yielded a strong association with having IIM in the dataset used to develop the criteria. ${ }^{8}$ In a previous response letter, we have discussed the process leading up to the final criteria and the selection process involving ILD and other extramuscular manifestations. ${ }^{9}$ From our local experience with the Karolinska University Hospital IIM cohort, out of our
14 patients with a positive anti-MDA5 autoantibody, 13 (93\%) are classifiable using the new classification criteria as either $\operatorname{DM}(n=9)$ or ADM $(n=4)$. Only one patient is not classifiable as he was diagnosed while critically ill with RPILD in the intensive care unit and died shortly after diagnosis, not unlike the case described by Lake et al. In that regard, we believe that most IIM classification criteria are performing poorly in an acute setting such as described above, given the challenge of assessing for muscle involvement by manual testing or muscle biopsy. We believe that in the example presented by Lake et al, the question is not if the 2017 EULAR/ACR IIM classification criteria are capturing or not anti-MDA5 cases, as they do. The question is if the subset assigned is an adequate reflection of the clinical phenotype associated with the condition. We must agree with the authors of the letter that in the current version of the EULAR/ACR IIM classification criteria, it is not possible to identify subgroups of rare phenotypes subclassified by MSA status. Our hope is that with time, international collaboration and expanding data will permit inclusion of other MSA in a data-driven validated revision of the EULAR/ACR IIM classification criteria.

\section{Valérie Leclair, ${ }^{1,2}$ Ingrid E Lundberg, ${ }^{1}$ Anna Tjärnlund ${ }^{1}$}

${ }^{1}$ Rheumatology Unit, Department of Medicine, Karolinska University Hospital, Karolinska Institutet, Stockholm, Sweden

${ }^{2}$ Rheumatology Unit, Department of Medicine, Jewish General Hospital, Montreal, Quebec, Canada

Correspondence to Anna Tjärnlund, Rheumatology Unit, Department of Medicine, Karolinska University Hospital, Karolinska Institutet, Stockholm, Sweden; anna.tjarnlund@ki.se

\section{Handling editor Josef S Smolen}

Funding The authors have not declared a specific grant for this research from any funding agency in the public, commercial or not-for-profit sectors.

Competing interests None declared.

Patient consent Not required.

Provenance and peer review Commissioned; internally peer reviewed.

(c) Article author(s) (or their employer(s) unless otherwise stated in the text of the article) 2019. All rights reserved. No commercial use is permitted unless otherwise expressly granted.

\section{Check for updates}

To cite Leclair V, Lundberg IE, Tjärnlund A. Ann Rheum Dis 2019;78:e53.

Received 16 April 2018

Accepted 17 April 2018

Published Online First 3 May 2018

\section{S Linked}

- http://dx.doi.org/10.1136/annrheumdis-2018-213285

Ann Rheum Dis 2019;78:e53. doi:10.1136/annrheumdis-2018-213519

\section{REFERENCES}

1 Lake M, George G, Summer R. Time to personalize the treatment of anti-MDA-5 associated lung disease. Ann Rheum Dis 2019;78:e52.

2 Lundberg IE, Tjärnlund A, Bottai M, et al. 2017 European League Against Rheumatism/ American College of Rheumatology classification criteria for adult and juvenile idiopathic inflammatory myopathies and their major subgroups. Ann Rheum Dis 2017;76:1955-64

3 Gono T, Kawaguchi Y, Satoh T, et al. Clinical manifestation and prognostic factor in anti-melanoma differentiation-associated gene 5 antibody-associated interstitial lung disease as a complication of dermatomyositis. Rheumatology 2010;49:1713-9.

4 Hall JC, Casciola-Rosen L, Samedy LA, et al. Anti-melanoma differentiation-associated protein 5-associated dermatomyositis: expanding the clinical spectrum. Arthritis Care Res 2013;65:1307-15. 
5 Malaviya AN. 2017 EULAR/ACR classification criteria for adult and juvenile idiopathic inflammatory myopathies and their major subgroups: little emphasis on autoantibodies, why? Ann Rheum Dis 2019;77:e77.

6 Lundberg IE, Tjärnlund A. Response to: '2017 EULAR/ACR classification criteria for adult and juvenile idiopathic inflammatory myopathies and their major subgroups: little emphasis on autoantibodies, why?' by Malaviya. Ann Rheum Dis 2018;77:e78.

7 Aggarwal R, Ringold S, Khanna D, et al. Distinctions between diagnostic and classification criteria? Arthritis Care Res 2015;67:891-7.
8 Bottai M, Tjärnlund A, Santoni G, et al. EULAR/ACR classification criteria for adult and juvenile idiopathic inflammatory myopathies and their major subgroups: a methodology report. RMD Open 2017;3:e000507.

9 Tjärnlund A, Bottai M, Lundberg IE. Response to Comments on the "2017 EULAR/ACR Classification Criteria for Adult and Juvenile Idiopathic Inflammatory Myopathies and Their Major Subgroups". Points of concern. Arthritis Rheumatol 2018. [Epub ahead of print 8 Mar 2018]. 